\title{
Visual encoding of patterns is subject to dual-task interference
}

\author{
ROBERTO DELL'ACQUA \\ University of Padova, Padua, Italy \\ and \\ PIERRE JOLICOEUR \\ University of Waterloo, Waterloo, Ontario, Canada
}

\begin{abstract}
Two stimuli were presented at varying stimulus onset asynchronies (SOAs), with each stimulus associated with a distinct task. The first stimulus was a tone at one of either two or three frequencies. In two conditions, the task associated with a tone was either a speeded two-alternative discrimination (2AD), or a speeded three-alternative discrimination (3AD) based on the pitch of the tone. In a third condition, subjects were told to ignore the tone. The second stimulus was a briefly exposed study matrix of red and black squares followed by a mask. After a fixed delay, the mask was replaced by a test matrix that was either the same or different from the study matrix. The task associated with the matrices was to indicate, with no speed pressure, whether the study matrix and the test matrix were the same or different. Results from each speeded $\mathrm{AD}$ condition showed that subject's accuracy in the matrix task decreased as the SOA between the tone and the study matrix decreased. This effect was larger for the $3 \mathrm{AD}$ tone task than with a $2 \mathrm{AD}$ tone task. In addition, within each speeded $\mathrm{AD}$ condition, longer RTs in the tone task were associated with lower accuracy in the matrix task. None of these effects was evident when the subjects were told to ignore the tone. These results suggest that encoding visual information can be subject to significant capacity limitations imposed by cross-modal multitasking.
\end{abstract}

Recent work suggests that under conditions of sequential presentation of a series of stimuli, the encoding of visual information from brief masked displays is subject to capacity limitations. These limitations have been described for within-modality dual-task paradigms (Broadbent \& Broadbent, 1987; Chun, 1997; Chun \& Potter, 1995; Duncan, Martens, \& Ward, 1997; Duncan, Ward, \& Shapiro, 1994; Jolicoeur, 1999b; Moore, Egeth, Berglan, \& Luck, 1996; Raymond, Shapiro, \& Arnell, 1992, 1995; Ward, Duncan, \& Shapiro, 1996, 1997; Weichselgartner \& Sperling, 1987).

Similar limitations have also been observed in betweenmodality dual-task paradigms (De Jong \& Sweet, 1994; Jolicoeur, 1999a, 1999b). Typically in these paradigms, each trial consists of the presentation of a tone at one of a number of different frequencies, followed by the presentation of a visual stimulus at variable stimulus onset asynchronies (SOAs). The task associated with the tone requires a speeded discrimination based on the pitch of the tone, and the task associated with the visual stimulus requires encoding. In De Jong and Sweet's experiments, the encoding task required the delayed report of the highest

This work was supported by a postdoctoral grant awarded to R. Dell'Acqua by Fondation Fyssen. The authors are indebted to Carlo Umiltà, John Wixted, Harold Pashler, Robert Ward, and one anonymous reviewer for helpful suggestions on a previous version of the present article. Address correspondence to R. Dell'Acqua, Department of Psychology, 8 Via Venezia, 35100 Padua, Italy (e-mail: dellacqu@psy.unipd.it). digit in an array of briefly presented masked digits. In Jolicoeur's (1999a, 1999b) experiments, the encoding task required the delayed report of briefly presented masked letters, or a delayed recognition of briefly presented masked polygons. The logic behind these paradigms is as follows: On the assumption that the speeded auditory discrimination task requires central mechanisms (Pashler, 1994a, 1998; Van Selst \& Jolicoeur, 1997), lower performance in the encoding task would be expected if some critical stage of encoding also required the same class of mechanisms. Accuracy in the encoding task should decrease as the SOA between the auditory and the visual stimulus was reduced, because the probability of contention for central mechanisms should increase as the process that mediated each task would overlap to a greater extent. De Jong and Sweet and Jolicoeur $(1999 \mathrm{a}, 1999 \mathrm{~b})$ found that accuracy in the visual encoding task decreased as the SOA between the tone and the to-be-encoded visual stimulus decreased, suggesting that encoding requires central mechanisms.

These recent findings, however, seem to conflict with earlier work that had provided support for the hypothesis that, in between-modality tasks, visual input is not subject to central capacity limitations. Specifically, Pashler (1993) reported near-nil effects of the SOA on the accuracy of recognizing a briefly presented masked matrix of squares in a paradigm formally equivalent to that used by Jolicoeur (1999a).

A number of important differences between Pashler's (1993) and Jolicoeur's (1999a) paradigms, however, may 
account for this specific empirical discrepancy. Perhaps encoding the spatial information that is required in order to perform the matrix-judgment task used by Pashler (1993) relied on a different form of memory than that required to remember visually presented letters (Jolicoeur, 1999a, Experiments 2 and 4), or the shape of a polygon (Jolicoeur, 1999a, Experiment 5). In this view, one might argue that encoding spatial information about the structure of a matrix does not require the same central mechanisms that appear to be involved in the encoding of visual form information (letters or polygons). Furthermore, another difference involves the relative difficulty of the tone task in these two studies. Pashler (1993) used a two-alternative discrimination (2AD) tone task, whereas Jolicoeur (1999a) used a $4 \mathrm{AD}$ tone task when polygons were to be remembered. It is likely that the $4 \mathrm{AD}$ tone task used by Jolicoeur (1999a, Experiment 5) taxed central mechanisms more than did the 2AD task used by Pashler (1993), with the consequence that the SOA effects on encoding accuracy were evident in Jolicoeur's, but not in Pashler's (1993) results.

Given the theoretical importance of processing requirements for visual encoding, a closer examination of the work of Pashler (1993) was warranted. The first experiment was an exact replication of Pashler's (1993) study. In the second experiment, a between-subjects manipulation was included to extended the original design. The number of alternatives in the speeded task was manipulated. One group of subjects performed a $2 \mathrm{AD}$ tone task, whereas a different group of subjects performed a $3 \mathrm{AD}$ tone task. The purpose of this manipulation was to discover whether performance in the matrix encoding task would be sensitive to the difficulty of the concurrent speeded tone task. The predictions were straightforward. If, as suggested by Pashler (1993), the matrix task relies on a memory system that does not require central processing capacity, we should be able to replicate the finding of a near-nil SOA effect on the matrix task accuracy in both experiments. If, in contrast, central mechanisms are required in the matrix task, accuracy should systematically decrease as the SOA is reduced. Furthermore, on the assumption that central processing mechanisms are engaged for a longer period of time in the 3AD task than in the 2AD task (Van Selst \& Jolicoeur, 1997), a more pronounced effect of SOA should occur in the 3AD task than in the $2 \mathrm{AD}$ task, when processing across the tone task and the matrix encoding task more likely overlap (i.e., at shorter SOAs).

\section{EXPERIMENT 1}

\section{Method}

Subjects. Thirteen students ( 7 women and 6 men) at the University of Padua volunteered to participate for pay or for course credit. Most of them were undergraduate students; a few were graduate students or staff. The ages of the subjects ranged from 20 to 31 years. All were naive to the purpose of the experiment, and all reported having normal or corrected-to-normal vision and normal hearing.

Auditory stimuli. The auditory stimuli were pure tones, presented for $150 \mathrm{msec}$, with a frequency of $300 \mathrm{~Hz}$ or $900 \mathrm{~Hz}$. The tones were presented through the speaker on the monitor of the computer and were well above threshold.

Visual stimuli. The visual stimuli consisted of random $4 \times 4$ matrices of black and red squares presented on a black background on a color computer screen (CRT) controlled by a $586 \mathrm{CPU}$ and MEL software. At a distance of $60 \mathrm{~cm}$, the side of each matrix measured approximately $5^{\circ}$ of visual angle. Each square in one matrix was black or red with chance probability. The RGB values were set at 0 , 0,0 for the black palette and at $40,0,0$ for the red palette. The CIE coordinates for the red color were $Y=5.15, x=.610, y=.360$.

Procedure. The sequence of the critical events on each trial is schematized in Figure 1. Each trial was initiated by the press of the spacebar on the computer keyboard. A fixation cross that was present between trials was replaced by a blank screen $(400 \mathrm{msec})$. A tone was then presented, and the task on the tone (Task 1 ) required subjects to perform a speeded 2AD based on the pitch of the tone. To do so, the subjects kept their index and middle fingers of the right hand on two appropriately labeled keys of a response box, and were instructed to treat Task 1 as their primary task. After an SOA of 50, 150 , or $650 \mathrm{msec}$, a study matrix was presented (red color represented by shaded areas in Figure 1). The study matrix was exposed for either 100 or $300 \mathrm{msec}$. At the end of the exposure duration, a mask composed of a $4 \times 4$ checkerboard pattern of black and red squares was presented for $100 \mathrm{msec}$. After mask offset, a fixed interval of $500 \mathrm{msec}$ elapsed before the presentation of a test matrix. The test matrix was exposed until the subject responded (see below). ${ }^{1}$

On half of the trials, the study matrix and the test matrix were the same; otherwise, the color of one square in the test matrix was randomly changed from red to black, or vice versa. The task on the matrices (Task 2) required an unspeeded decision in order to indicate

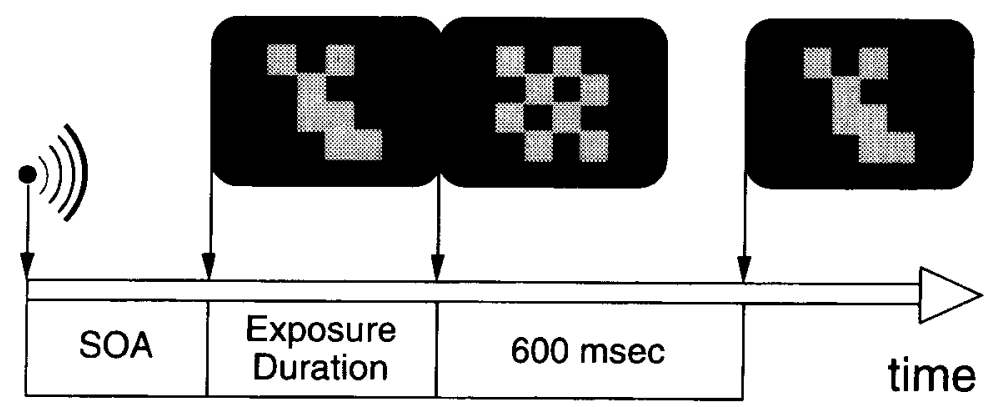

Figure 1. Schematic representation of the temporal sequence of the events on each trial of Experiment 1 (see also Pashler, 1993). 
whether the study matrix and the test matrix were the same or different. Using the numeric keypad, the subjects had to press the " 1 " to respond "same," or " 2 " to respond "different." Each subject was tested individually in a separate room. The experiment began with 2 blocks of 12 practice trials each, followed by 12 blocks of 24 experimental trials each. Tone pitch, SOA, exposure duration of the study matrix, and response category (same/different) levels of manipulation were fully crossed within each block of trials.

Method of analysis. In the present paradigm, Task 1 required the speeded response, and the reaction time (RT1) was recorded. The correct RT1s were screened for outliers with a modification of the procedure described by Van Selst and Jolicoeur (1994). The data in each cell were sorted, and the most extreme observation was temporarily excluded from consideration. The mean and standard deviation of the remaining numbers was then computed. The cut-off values were established with the use of following equations:

$$
\begin{aligned}
& V_{\text {low }}=X-\mathrm{Cn} * S D \\
& V_{\text {high }}=X+\mathrm{Cn} * S D .
\end{aligned}
$$

The smallest and largest observations in the cell were then checked against the cut-off values, $V_{\text {low }}$ and $V_{\text {high. }}$. If one or both were outside the bounds, these observations were defined as outliers and excluded from further consideration. If an outlier was found, the algorithm was applied anew to the remaining data. The value of $\mathrm{Cn}$ depended on the sample size, $n$, in such a way that the estimated final mean was not influenced by sample size (see Van Selst \& Jolicoeur, 1994). This procedure resulted in a loss of less than $1.8 \%$ of the correct trials. When an outlier (or an error) in the speeded Task 1 was found, the entire trial was discarded, including the data for Task 2.

\section{Results}

The results from both Task 1 and Task 2 were analyzed using an analysis of variance (ANOVA) in which SOA and study matrix exposure duration were treated as withinsubjects factors.

Task 2. In panel A of Figure 2, the proportion of correct matrix judgments is shown as a function of the SOA between the tone and the study matrix, and as a function of the exposure duration of the study matrix. Accuracy in Task 2 decreased as the SOA was shortened $[F(2,24)=$ $\left.9.8, M S_{\mathrm{e}}=.003215, p<.001\right]$, and accuracy was higher at the longer exposure duration $(300 \mathrm{msec})$ than at the shorter exposure duration $(100 \mathrm{msec})$ of the study matrix $\left[F(2,24)=269.3, M S_{\mathrm{e}}=.004619, p<.001\right]$. The interaction between SOA and exposure duration of the study matrix was not significant $(F<1, p>.9) .^{2}$

Accuracy in Task 2 was also analyzed as a function of the speed in Task 1. Following outlier screening of the data set, the proportion of correct matrix judgments was computed for each subject, in each cell of the present design, for RT1s divided into four bins based on a quartile split. In panel B of Figure 2, the proportion of correct matrix judgments is plotted as a function of the SOA between the tone and the study matrix, and as a function of RT1 length $(\mathrm{Q} 1=$ shortest RT1s; Q4 = longest RT1s). Accuracy decreased systematically across $\mathrm{RT} 1$ quartiles as RT1 s increased $\left[F(3,36)=15.7, M S_{\mathrm{e}}=.014164, p<.001\right]$. Notably, SOA effects on accuracy were more pronounced as RT 1s increased $\left[F(6,72)=2.7, M S_{\mathrm{e}}=.016586, p<.03\right]$. This interaction appears to have been due primarily to an
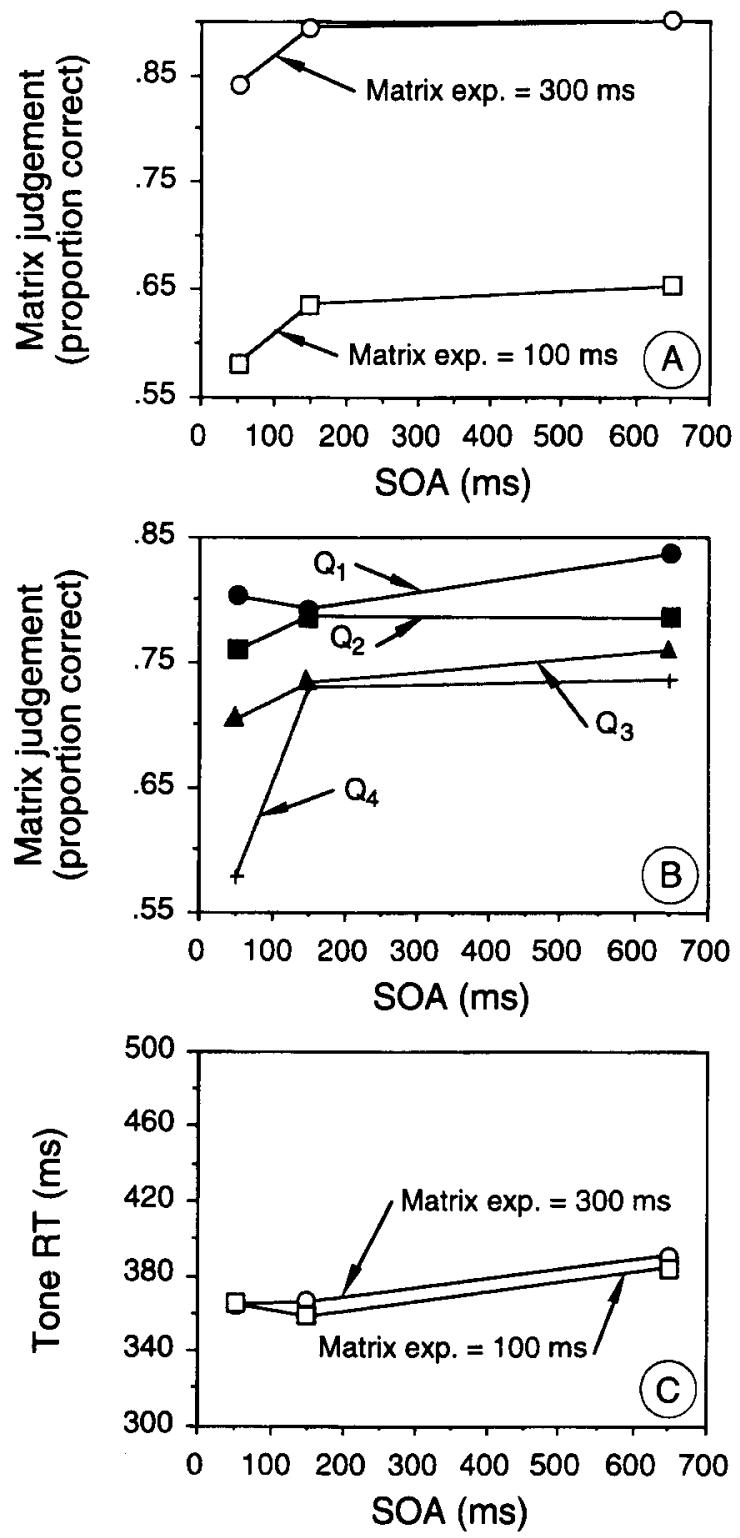

Figure 2. (A) Mean proportion of correct matrix judgments (Task 2) as a function of the SOA between the tone and the study matrix, and as a function of the exposure duration of the study matrix (100 msec vs. $300 \mathrm{msec}$ ). (B) Mean proportion of correct matrix judgments (Task 2) as a function of the SOA between the tone and the study matrix, and as a function of RT length in Task 1 (Q1 = shortest RT; Q4 = longest RT). (C) Mean tone RT (Task 1) as a function of the SOA between the tone and the study matrix, and as a function of the exposure duration of the study matrix.

increase in the size of the SOA effect for the longest response times in Task 1.

Task 1. RT1s and Task 1 accuracy were analyzed as a function of the same variables considered for the analysis of Task 2 accuracy. In panel C of Figure 2, RT $1 \mathrm{~s}$ are shown as a function of the SOA between the tone and the study matrix, and as a function of the study matrix exposure du- 
ration. A 20-msec increase in RT1s from the two shorter SOAs to the longest SOA produced a significant effect of SOA $\left[F(2,24)=9.15, M S_{\mathrm{e}}=498, p<.002\right]$. Mean comparisons revealed that RT $1 s$ at the two shorter SOAs did not differ significantly $(F<1, p>.6)$. No other effect was significant $(F<1, p>.35$, in all cases).

The mean proportion of correct responses in Task 1 was .972. The proportion of correct responses was higher at the longest SOA (.983) than at the shortest and medium SOAs (.967 and .966 , respectively) $\left[F(2,24)=5.4, M S_{\mathrm{e}}=\right.$ $.000438, p<.02]$. Mean comparisons indicated that the proportion of correct responses did not differ significantly at the two latter SOAs $[F(1,12)<1, p>.9]$. No other effect was significant $(F<1.2, p>.36$, in all cases).

\section{Discussion}

Accuracy in the matrix task decreased significantly as the SOA between the tone and the study matrix was reduced. The results are consistent with the hypothesis that encoding the information that is required in order to perform the matrix task relies on central mechanisms that are subject to interference from the concurrent processing of a stimulus in a different modality. In addition to the effects of SOA, accuracy in the matrix task was also found to decrease as response times in Task 1 increased. This finding is also consistent with the hypothesis that the two tasks interfere with each other (Pashler, 1994a). In the General Discussion we will discuss the discrepancy between the magnitude of the SOA effect in Experiment $1(7 \%)$ and the effect reported by Pashler (1993) of $2 \%$.

\section{EXPERIMENT 2}

The dual-task interference found in Experiment 1 is consistent with the hypothesis that the matrix task requires capacity-limited central processing. Experiment 2 was designed in order to provide converging evidence for this hypothesis. The number of alternatives in the concurrent speeded choice task was manipulated. Previous work has shown that a longer period of central processing is associated with speeded choice tasks that have more response alternatives (e.g., Van Selst \& Jolicoeur, 1997). If the matrix encoding task requires central processing, the effects of SOA should be larger when the concurrent choice task engages central processing for a longer period of timethat is, when the task has more response alternatives (Jolicoeur, 1999a). The tone task involved either two tones and two responses (2AD), or three tones and three responses (3AD), which should vary the duration of central processing required in order to perform the tone task. Furthermore, in order to span a longer period of tasks interaction, we added a longer SOA of $950 \mathrm{msec}$ to the original set of SOAs that were used by Pashler (1993) and in our Experiment 1.

\section{Method}

Subjects. Thirty-six students ( 16 women, 20 men) at the University of Padua volunteered to participate for pay or for course credit. Most of them were undergraduate students; a few were graduate stu- dents or staff. The ages of the subjects ranged from 20 to 33 years. All were naive to the purpose of the experiment, and all reported having normal or corrected-to-normal vision and normal hearing. None of the subjects had participated in Experiment 1 .

Auditory stimuli. The auditory stimuli were pure tones, presented for $100 \mathrm{msec}$, with a frequency of 300,600 , or $900 \mathrm{~Hz}$. The tones were presented through the speaker on the monitor of the computer and were well above threshold.

Visual stimuli. The visual stimuli were the same as those employed in Experiment 1.

Procedure. Each trial was initiated by the press of the spacebar on the computer keyboard. A fixation cross that was present between trials was replaced by a blank screen $(400 \mathrm{msec})$. A tone was then presented, and the task associated with the tone (Task 1) required a speeded discrimination based on the pitch of the tone. Half of the subjects performed a $2 \mathrm{AD}$ task that was based on a pitch of either 300 or $900 \mathrm{~Hz}$, whereas the other half of the subjects performed $3 \mathrm{AD}$ task that was based on a pitch of either 300,600 , or $900 \mathrm{~Hz}$. Depending on the AD condition, the subjects kept either two or three fingers of the right hand on the appropriately labeled keys of the response box and were instructed to treat Task 1 as their primary task. After an SOA of $50,150,650$, or $950 \mathrm{msec}$, a study matrix was presented for $170 \mathrm{msec}$. At the end of the exposure duration, a mask composed of a $4 \times 4$ checkerboard pattern of black and red squares was presented for $100 \mathrm{msec}$. After mask offset, a fixed interval of $680 \mathrm{msec}$ elapsed before the presentation of a test matrix. The test matrix was exposed until the subject responded (see below). On half of the trials, the study matrix and the test matrix were the same; otherwise, the color of one square in the test matrix was randomly changed from red to black, or vice versa. The task associated with the matrices (Task 2) and the instructions given to the subjects were the same as those in Experiment 1.

Each subject was tested individually in a separate room. The experiment began with two blocks of practice trials. In the $2 \mathrm{AD}$ condition, the subjects performed a total of 24 practice trials, followed by six blocks of 32 experimental trials each. In the $3 \mathrm{AD}$ condition, the subjects performed a total of 48 practice trials, followed by seven blocks of 24 experimental trials each. In each AD condition, tone pitch, SOA, and response category (same/different) levels of manipulation were fully crossed within each block of experimental trials.

\section{Results}

The correct RT1s were screened for outliers according to the procedure that was described in Experiment 1. Elimination of outliers produced a total loss of $1.5 \%$ for Task 1 and Task 2 responses. The results from both Task 1 and Task 2 were subjected to an ANOVA, in which the AD condition was treated as a between-subjects factor, and the SOA as a within-subjects factor.

Task 2. In panel A of Figure 3, the proportion of correct matrix judgments (solid lines and empty symbols) is shown as a function of the SOA between the tone and the study matrix, and as a function of the AD condition. Accuracy in Task 2 decreased as the SOA was shortened $\left[F(3,102)=53.4, M S_{\mathrm{e}}=.004909, p<.001\right]$, and this effect was more pronounced in the $3 \mathrm{AD}$ condition than in the $2 \mathrm{AD}$ condition $\left[F(3,102)=5.6, M S_{\mathrm{e}}=.004909, p<.002\right]$.

Accuracy in Task 2 was also analyzed as a function of the speed in Task 1. Following outlier screening of the data set, the proportion of correct matrix judgments was computed for each subject, in each cell, for RT $1 \mathrm{~s}$ above or below the median in that cell. In panel B of Figure 3, the proportion of correct matrix judgments is plotted as a function of the SOA between the tone and the study ma- 

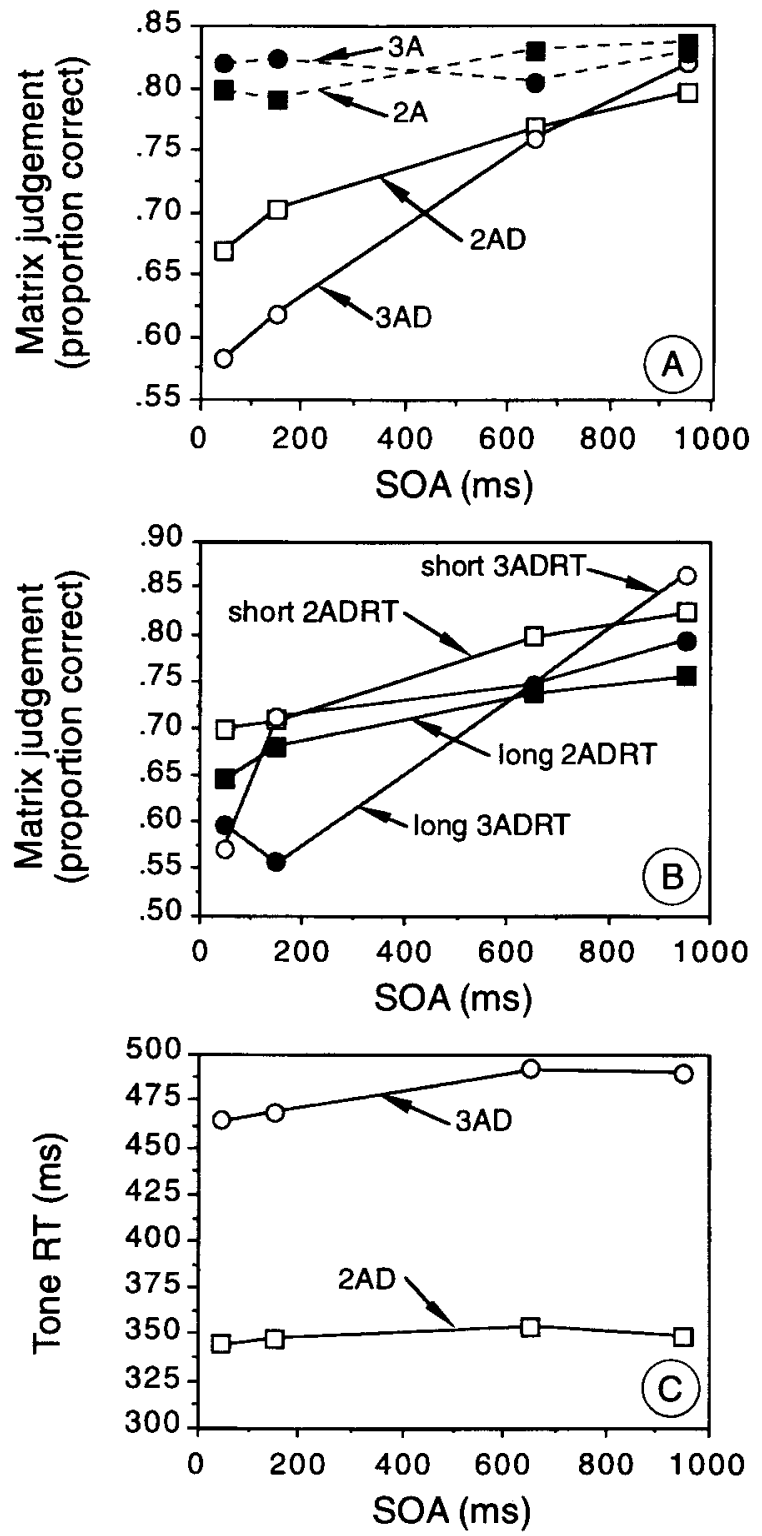

Figure 3. (A) Solid lines and empty symbols (Experiment 2): Mean proportion of correct matrix judgments (Task 2) as a function of the SOA between the tone and the study matrix, and as a function of Task $1 \mathrm{AD}$ condition (2AD vs. 3AD). Dashed lines and filled symbols (Experiment 3): Mean proportion of correct matrix judgments as a function of the SOA between the tone and the study matrix, and as a function of the number of pitch alternatives (2A vs. 3A). (B) Mean proportion of correct matrix judgments (Task 2) in Experiment 2, as a function of the SOA between the tone and the study matrix, and as a function of RT length (short vs. long) in Task 1. (C) Mean tone RT (Task 1) in Experiment 2 as a function of the SOA between the tone and the study matrix, and as a function of Task $1 \mathrm{AD}$ condition.

trix, of RT1 length, and of AD condition. Accuracy in Task 2 was higher with shorter RT 1s than with longer RT 1s $\left[F(1,34)=24.9, M S_{\mathrm{e}}=.009176, p<.001\right]$. The analysis revealed a significant three-way interaction between RT length, $\mathrm{SOA}$, and $\mathrm{AD}$ condition $\left[F(3,102)=3.7, M S_{\mathrm{e}}=\right.$
$.009635, p<.02]$. To elucidate the source of this complex interaction, separate analyses were carried out on each of the data sets from the different $\mathrm{AD}$ conditions. The analysis carried out on the data set from the $3 \mathrm{AD}$ condition revealed a significant main effect of RT length $[F(1,17)=$ $\left.10.8, M S_{\mathrm{e}}=.013822, p<.005\right]$ and a significant interaction between RT length and SOA $\left[F(3,51)=4.8, M S_{\mathrm{e}}=\right.$ $.010974, p<.004]$. The analysis carried out on the data set from the $2 \mathrm{AD}$ condition, in contrast, revealed a significant main effect of RT length $\left[F(1,17)=18.3, M S_{\mathrm{e}}=.004529\right.$, $p<.001]$ and no significant interaction between RT length and SOA $(F<1, p>.75)$. As can be seen in panel b of Figure 3, in fact, although the SOA functions were parallel across RT1s in the $2 \mathrm{AD}$ condition, the SOA functions for longer RT $1 \mathrm{~s}$ and for shorter RTls in the 3AD condition were, respectively, positively and negatively accelerated.

Task 1. RT1s and Task 1 accuracy were analyzed as a function of the same variables considered for the analysis of Task 2 accuracy. In panel C of Figure 3, RT 1s are shown as a function of the SOA between the tone and the study matrix and as a function of the AD condition. RT 1s were longer in the $3 \mathrm{AD}$ condition than in the $2 \mathrm{AD}$ condition $\left[F(1,34)=26.5, M S_{\mathrm{e}}=22,890, p<.001\right]$, and RT 1s increased slightly from the two shorter SOAs to the two longer $\operatorname{SOAs}\left[F(3,102)=7.2, M S_{\mathrm{e}}=374, p<.001\right]$. There was also a significant interaction between SOA and AD condition $\left[F(3,102)=3.1, M S_{\mathrm{e}}=374, p<.03\right]$, which likely reflected the fact that, as panel $\mathrm{C}$ of Figure 3 suggests, SOA effects were confined to the $3 \mathrm{AD}$ condition. To provide support for this impression, the data sets from each condition were separately analyzed. A significant main effect of SOA was found in the analysis carried out on the data set from the $3 \mathrm{AD}$ condition $\left[F(3,51)=7.5, M S_{\mathrm{e}}=\right.$ $483, p<.001]$. SOA effects were not significant in the analysis carried out on the data set from the $2 \mathrm{AD}$ condition $(F<1, p>.45)$.

The mean proportion of correct responses in Task 1 was .891. The proportion of correct responses was higher in the $2 \mathrm{AD}$ condition (.939) than in the $3 \mathrm{AD}$ condition $(.836)$ $\left[F(1,34)=13.8, M S_{\mathrm{e}}=.028185, p<.001\right]$. The proportion of correct responses increased as SOA was lengthened $(.860, .891, .899$, and .901 , from the shortest to the longest SOA, respectively) $\left[F(3,102)=6.0, M S_{\mathrm{e}}=.002228, p<\right.$ $.001]$. The interaction between SOA and the AD condition was not significant $(F<1, p>.55)$.

\section{Discussion}

In Experiment 2, the number of alternatives in the concurrent speeded choice task was manipulated. At short SOAs, the Task 1 manipulation caused a greater decrease in Task 2 accuracy when the number of alternatives was increased. On the assumption that a longer period of central processing is associated with speeded choice tasks that have more response alternatives (see, e.g., Van Selst \& Jolicoeur, 1997), our claim is that the results of Experiment 2 provide strong evidence for the hypothesis that performance on the matrix task is subject to central limitations. 


\section{EXPERIMENT 3}

In our view, the effects of SOA on the matrix task are due to central processing that is required for one to perform the tone task. One could argue, however, that the alerting properties of the tone (see, e.g., Posner, 1978) were confounded with other possible effects of SOA. Although this account provides no obvious explanation for the large difference between the two-alternative and threealternative versions of the tone task, we conducted Experiment 3 in order to provide a strong case against interpretations based on alerting properties of the tone. If interference on the matrix task was mediated by the warning function of the tone, the same pattern of interference found in Experiments 1 and 2 should be found even if the subjects were not required to produce an overt response to the tone. On the other hand, if the effects of SOA were due to central processing in the tone task, effects of SOA should vanish when the tone was not processed.

\section{Method}

Subjects. Thirteen students ( 6 women, 7 men) at the University of Padua volunteered to participate for pay or for course credit. Most of them were undergraduate students; a few were graduate students or staff. The ages of the subjects ranged from 20 to 30 years. All were naive to the purpose of the experiment, and all reported having normal or corrected-to-normal vision and normal hearing. None of the the subjects had participated in Experiment 1 or 2.

Auditory stimuli. The auditory stimuli were the same as those used in Experiment 2.

Visual stimuli. The visual stimuli were the same as those used in Experiments 1 and 2.

Procedure. The sequence and the duration of the events on each trial were the same as those in Experiment 2. In separate blocks, the tone pitch would randomly vary between two $(300 \mathrm{or} 900 \mathrm{~Hz})$ or among three possible pitches $(300,600$, or $900 \mathrm{~Hz})$. After a varying SOA, a masked study matrix was presented, followed by presentation of a test matrix. The subjects were instructed to ignore the tone, and to perform the same/different judgment on the matrices with no speed requirement. The experiment began with one block of 24 practice trials, followed by four blocks of 48 experimental trials each: two blocks in which the tone had two possible pitches, and two blocks in which the tone had three possible pitches. Block order was randomized for each subject. Number of tone pitches, SOA, and response category (same/different) levels of manipulation were fully crossed within each block of experimental trials.

\section{Results}

The results from 1 subject were discarded because the subject did not understand the instructions. Mean proportions of correct matrix judgments were subjected to an ANOVA, in which number of pitches and SOA were treated as within-subjects factors. Overall, the mean proportion of correct matrix judgments was .82. In panel a of Figure 3 , the proportion of correct matrix judgments (dashed lines and filled symbols) is shown as a function of the SOA between the tone and the study matrix, and as a function of the number of tone pitches ( $2 \mathrm{~A}$ vs. $3 \mathrm{~A})$. No factor reached the significance level $(F<1$, and $p>.53$, in all cases).

\section{GENERAL DISCUSSION}

Conflicting results have been reported regarding whether or not the encoding of visual information is affected by the temporal overlap with unrelated cognitive activity. In a dual-task paradigm that involved a speeded auditory task and an unspeeded task that required visual encoding, Jolicoeur (1999a), on the one hand, reported a significant decrease in recognition accuracy as the SOA between the tone and a masked random polygon was decreased. Pashler (1993), on the other hand, reported no such effect when the SOA between the tone and a masked random matrix was decreased.

In order to discover the cause of this apparent empirical discrepancy, Experiment 1 was devised to replicate the experiment reported by Pashler (1993). In contrast to the near-nil (2\%) effect of the SOA (not statistically significant) found by Pashler (1993), the results of Experiment 1 indicated a $7 \%$ decrease in accuracy in the matrix task, which was highly statistically significant, as the SOA between the tone and the study matrix decreased. Also found was clear-cut evidence for trial-to-trial dependencies across tasks, in which worse performance in the matrix task was associated with longer RTs to the tone. These dependencies were more pronounced as the SOA was decreased. Similar dependencies were also found in Experiment 2. These findings also contrast with Pashler's (1993) results. Besides the small SOA effects in the results of his experiment, Pashler (1993) also argued against the involvement of central mechanisms in the encoding of the study matrix on the basis of a comparison between effects of SOA and effects of exposure duration. Pashler observed that a reduction in exposure duration of the study matrix of $200 \mathrm{msec}$ produced a $15 \%$ decrease in the matrix task accuracy. In our Experiment 1, the exposure duration effect was about $20 \%$. If the size of the exposure duration effect is taken as a benchmark against which to estimate the effects of different time constraints on the matrix task, the conclusion must be that the constraint provided by the temporal overlap between the speeded task and the matrix task was truly minimal, as Pashler (1993) argued. (To reiterate, from the longest to the shortest SOA, a $2 \%$ decrease in accuracy was observed in Pashler's experiment, whereas a 7\% decrease in accuracy was observed in our Experiment 1). There is a problem, however, with this line of argument. As suggested by the additivity of SOA and exposure duration effects in both Pashler's (1993) experiment and in our Experiment 1, exposure duration and SOA may affect distinct stages of processing. Furthermore, interference (such as postponement) at these different stages could have different effects on the final matrix task accuracy. Although it seems reasonable to maintain that a reduction of the exposure duration of a visual pattern affects a sensory stage of processing, recent work in our labs suggests that the stage that is subject to central limitations is a postsensory stage of processing - namely, the short-term consolidation of information into short-term memory (Jolicoeur, 
1999a; Jolicoeur \& Dell'Acqua, 1998, 1999, in press; see also Chun \& Potter, 1995). In this view, exposure duration effects hinge on a reduction of the time that is required for the creation of a visual sensory trace. Effects of SOA, however, would occur later in the system, because of a delay in the onset of short-term consolidation. During this period of delay, information created by sensory processing would be subject to decay. The rate of decay could be considerably slower than the rate at which sensory information accrues, leading to quite different relationships between the effects associated with a given duration of postponement and the effects associated with the duration of visual stimulation (prior to the onset of a mask).

In Experiment 2, we manipulated the number of response alternatives in Task 1. Van Selst and Jolicoeur (1997) provided evidence that when the number of stimulus and response alternatives is manipulated in Task 2 of a PRP (psychological refractory period) paradigm, the effects of this manipulation remain stable across SOAs, thus suggesting that the locus of the effect is in or after the stage (or stages) of processing that produces the interference in the PRP paradigm. Indeed, several findings suggest that a Task 2 manipulation that affects a stage prior to the PRP bottleneck (e.g., a perceptual stage) likely results in an underadditive interaction with SOA, as SOA is shortened (De Jong, 1993; McCann \& Johnston, 1992; Pashler, 1994b). Although both experiments in the present study suggest that visual encoding is subject to capacity limitations, the results of Experiment 2 are especially compelling because a manipulation that is believed to affect the duration of central processing in Task 1 (Van Selst \& Jolicoeur, 1997) caused an increase in the magnitude of the dual-task interference effect observed in Task 2 accuracy. This evidence provides support for the hypothesis that encoding visual information is subject to interference from concurrent cognitive activity that is required in order to perform a speeded auditory task (Jolicoeur \& Dell'Acqua, 1998, 1999), even when the visual display consists of a random matrix, rather than letters or random polygons (Jolicoeur, 1999a). Thus, the hypothesis that the empirical differences across Pashler's (1993) and Jolicoeur's (1999a) results might be attributed to a difference in stimulus materials (matrices vs. random polygons) was not supported. Furthermore, our interpretation of the present findings is also supported by the results of our Experiment 3, which was devised to rule out possible accounts based on alerting properties of the tone (i.e., the first stimulus) in our paradigms.

Because the results of Experiment 1, which was extremely similar to the experiment performed by Pashler (1993), provided evidence for dual-task interference, the results are difficult to reconcile with Pashler's suggestion that the processes that mediate the encoding of visual information into short-term memory are capacity free (Pashler, 1993; see also Pashler, 1994a, 1998). There is another difference between the results of Pashler (1993) and those in this article, however, that could provide the basis for an account of the differences in results. The mean response times in Task 1 reported by Pashler (1993) were almost 100 msec longer than those in the present experiments. This suggests that Pashler's subjects may have been less prepared for the auditory task than ours. Perhaps Pashler's subjects were trading off, on some proportion of trials, speed in the auditory task for accuracy in the demanding matrix encoding task (De Jong \& Sweet, 1994).

The difference in response times for the auditory task in Pashler's (1993) experiment and our Experiments 1 and 2 could have been caused by a greater emphasis on performing the auditory task quickly, on the part of our subjects. This possibility raises the issue of the role of preparatory versus structural limitations in performing concurrent tasks. Although strong evidence suggests that some dualtask limitations reflect a fundamental inability of a central channel to process more than one stimulus or task at a time (Pashler, 1994b), other evidence suggests that limitations can sometimes arise when central mechanisms, albeit potentially capable of some parallel processing, can be allocated to and/or prepared for only one discrete task at a time (De Jong, 1995; De Jong \& Sweet, 1994). Although the SOA effects consistently found in our experiments suggest some form of central limitation in the encoding of visual information, determining the exact nature of this limitation (whether a structural bottleneck, a form of task preparation, or both) will require additional research.

\section{REFERENCES}

Broadbent, D. E., \& Broadbent, M. H. P. (1987). From detection to identification: Response to multiple targets in rapid serial visual presentation. Perception \& Psychophysics, 42, 105-113.

ChUn, M. M. (1997). Types and tokens in visual processing: A double dissociation between the attentional blink and repetition blindness. Journal of Experimental Psychology: Human Perception \& Performance, 23, 738-755.

Chun, M. M., \& PotTer, M. C. (1995). A two-stage model for multiple target detection in rapid serial visual presentation. Journal of Experimental Psychology: Human Perception \& Performance, 21, 109-127.

DE JONG, R. (1993). Multiple bottlenecks in overlapping task performance. Journal of Experimental Psychology: Human Perception \& Performance, 19, 965-989.

DE JONG, R. (1995). The role of preparation in overlapping-task performance. Quarterly Journal of Experimental Psychology, 48A, 2-25.

DE JONG, R., \& SwEET, J. B. (1994). Preparatory strategies in overlappingtask performance. Perception \& Psychophysics, 55, 142-151.

DunCan, J., MarTens, S., \& Ward, R. (1997). Restricted attentional capacity within but not between sensory modalities. Nature, 387, 808810.

Duncan, J., Ward, R., \& Shapiro, K. L. (1994). Direct measurement of attentional dwell time in human vision. Nature, 369, 313-315.

Jolicoeur, P. (1999a). Dual-task interference and visual encoding. Journal of Experimental Psychology: Human Perception \& Performance, $25,630-648$.

JoLICOEUR, P. (1999b). Restricted attentional capacity between sensory modalities. Psychonomic Bulletin \& Review, 6, 87-92.

Jolicoeur, P., \& Dell'AcQua, R. (1998). The demonstration of shortterm consolidation. Cognitive Psychology, 36, 138-202.

Jolicoeur, P., \& Dell'Acqua, R. (1999). Attentional and structural constraints on visual encoding. Psychological Research, 62, 154-164. 
Jolicoeur, P., \& Dell'ACQua, R. (in press). Selective influence of second target exposure duration and Task 1 load effects in the attentional blink phenomenon. Psychonomic Bulletin \& Review.

MCCANN, R. S., \& Johnston, J. C. (1992). Locus of the single-channel bottleneck in dual-task interference. Journal of Experimental Psychology: Human Perception \& Performance, 18, 471-484.

Moore, C. M., Egeth, H., Berglan, L. R., \& Luck, S. J. (1996). Are attentional dwell times inconsistent with serial visual search? Psy. chonomic Bulletin \& Review, 3, 360-365.

Pashler, H. (1993). Dual-task interference and elementary mental mechanisms. In D. E. Meyer \& S. Kornblum (Eds.), Attention and performance XIV: Synergies in experimental psychology, artificial intelligence, and cognitive neuroscience (pp. 245-264). Cambridge, MA MIT Press.

PAShler, H. (1994a). Dual-task interference in simple tasks: Data and theory. Psychological Bulletin, 2, 220-244.

PASHLER, H. (1994b). Overlapping mental operations in serial performance with preview. Quarterly Journal of Experimental Psychology, 47A, 161-192.

Pashler, H. (1998). The psychology of attention. Cambridge: MIT Press.

PosNer, M. I. (1978). Chronometric explorations of mind. Hillsdale, NJ: Erlbaum.

Raymond, J. E., Shapiro, K. L., \& ARnell, K. M. (1992). Temporary suppression of visual processing in an RSVP task: An attentional blink? Journal of Experimental Psychology: Human Perception \& Performance, 18, 849-860.

Raymond, J. E., Shapiro, K. L., \& Arnell, K. M. (1995). Similarity determines the attentional blink. Journal of Experimental Psychology: Human Perception \& Performance, 21, 653-662.

VAN SELST, M., \& JolicoeuR, P. (1994). A solution to the effect of sample size on outlier elimination. Quarterly Journal of Experimental Psychology, 47A, 63I-650.

VAN SElst, M., \& Jolicoeur, P. (1997). Decision and response in dualtask interference. Cognitive Psychology, 33, 266-307.
Ward, R., Duncan, J., \& Shapiro, K. (1996). The slow time-course of visual attention. Cognitive Psychology, 30, 79-109.

Ward, R., DunCaN, J., \& Shapiro, K. (1997). Effects of similarity, difficulty, and nontarget presentation on the time course of visual attention. Perception \& Psychophysics, 59, 593-600.

Weichselgartner, E., \& SperLing, G. (1987). Dynamics of automatic and controlled visual attention. Science, 238, 778-780.

\section{NOTES}

\section{Instructions given to the subjects:}

On each trial of this experiment, you will see a fixation cross displayed in the center of the screen. In order to start each trial, you have to press the spacebar. After pressing the spacebar, you will hear a tone that could be high or low in pitch. Your primary task is to press the left key of the response box if the pitch is high, or the right key of the response box if the pitch is low. Always make your response to the tone as fast and accurate as you can. Following the tone, a matrix composed of red squares will be briefly displayed in the center of the screen. This matrix will be masked by a "checkerboard" matrix, and followed by another matrix, which will stay on the screen until you respond. Your task is to press "1" if the first matrix and the last matrix are identical, or " 2 " if the first matrix and the last matrix are different. The task with the matrices is not speeded: make your response with no speed pressure, and try to be as accurate as you can

2. In order to rule out accounts of the present findings that are based on possible criterion shifts across SOAs, all the analyses performed on the proportion of correct same/different judgments have also been performed using the same experimental designs and $d^{\prime}$ as the dependent measure. The results of the analyses on $d^{\prime}$ mirror and fully support the results reported in the empirical sections of the present work.

(Manuscript received January 8, 1999 ; accepted for publication March 3, 1999.) 